\title{
Measurements of the ion-depletion zone evolution in a micro/ nano-channel
}

\author{
Qun Yu $\cdot$ Zhanhua Silber-Li
}

Received: 11 March 2011/Accepted: 31 May 2011/Published online: 22 June 2011

(C) Springer-Verlag 2011

\begin{abstract}
Ion-depletion and ion-enrichment are the most important phenomena found in a hybrid micro/nano-channel. This depletion process significantly decreases the ion concentration at the anodic end of the nano-channels and lower concentration propagation in the microchannel. The present paper focuses on measurement and analyzes the extension of the depletion zone with time and propagation of concentration perturbation. The systemic experiments were conducted using the nano-porous membranes with different pore diameters $(\phi 15 \mathrm{~nm}, \phi 50 \mathrm{~nm})$, the applied electric voltages $(20-100 \mathrm{~V})$ and the buffer solution $\mathrm{pH}$ values ( $\mathrm{pH} 5.4,9.2)$. According to dimensionless analysis, the extension of the depletion boundary was initially found to be independent of time and to have a non-linear variation for larger times. Based on Nernst-Planck equation, a one-dimensional wave equation is obtained under certain hypothesis, which also shows the propagation of concentration perturbation in depletion zone with constant speed at the initial time.
\end{abstract}

Keywords Ion-depletion and enrichment . Depletion extension - Propagation velocity .

Hybrid micro/nano-channels

\section{Introduction}

The rapid development of nano-fabrication technology has allowed the application of nanofluidics to various new

Q. Yu $\cdot$ Z. Silber-Li ( $\square)$

State Key Laboratory of Nonlinear Mechanics,

Institute of Mechanics, Chinese Academy of Sciences,

Beijing 100190, China

e-mail: lili@imech.ac.cn fields. For example, they have been adapted for sample analysis (Garcia et al. 2005; Griffiths and Nilson 2006; Wang et al. 2006) and for use in the preparation of new chemical substances (Wang et al. 2005; Song and Singh 2006). Nano-fluidic devices exploit length scales on the order of the bio-molecular diameter or, equivalently, the thickness of the electrical double layers (EDLs) that form adjacent to charged surfaces. The electro-kinetic flow behavior in hybrid micro/nano-channels has been found to be very complex due to the interaction of the electric field with the charged ions and the electro-osmotic velocity field. Earlier studies have shown that the most important phenomena relate to the ion-enrichment and ion-depletion processes that are found to exist at the ends of the nanochannels (Pu et al. 2004; Plecis et al. 2005; Datta et al. 2006; Kim et al. 2007).

The development of the depletion zone with time was found to be important for the understanding of the enrichment/depletion mechanism. A significant body of literature has been devoted to the study of this depletion zone development. For example, Huang and Yang (2008) observed the final location of the depletion boundary under different voltages in a cross-form micro/nano-channel. Lee et al. (2008) also measured the size of the depletion zone as a function of voltage. Then, Yossifon and Chang (2008) studied the development of the depletion zone length $L$ outside a nanoslot without convection flow under AC voltage. They depicted the length of the depletion zone for different frequencies $(<0.2 \mathrm{~Hz})$, at the same voltage difference of $80 \mathrm{~V}_{\mathrm{p}-\mathrm{p}}$, and found that the length of the depletion zone for low-frequencies obeyed the simple $\sqrt{D t}$ scaling, where $D$ is the diffusion coefficient. Zangle et al. (2009) fabricated the two-dimensional hybrid channel containing a single nano-channel $(\phi 50-100 \mathrm{~nm}$ high, $20 \mu \mathrm{m}$ wide, 
$100 \mu \mathrm{m}$ long). They first mentioned the shock-wave happened in the depletion zone and observed the shock-wave velocity of the depletion boundary under constant current (50 pA to $16 \mathrm{nA}$ ), and showed that this velocity increased in proportion to the current density.

All the results noted above improve the understanding of the enrichment/depletion phenomenon, but the systemic experiments, considering the influences of external voltage, the nanopore size and $\mathrm{pH}$ value, have not been presented.

The study outlined herein focused upon the measurement of the depletion zone extension and the corresponding concentration change with time. The parameters influencing this development include the external voltage, the pore diameter of the nanoporous membrane and the $\mathrm{pH}$ values of the buffer solution. The systemic experiments carried out under an applied electric field with voltage in the range $25-100 \mathrm{~V}$, the nanopore sizes $(\phi 15 \mathrm{~nm}, \phi 50 \mathrm{~nm})$ and the buffer solution $\mathrm{pH}$ values $(\mathrm{pH} 5.4$ and 9.2). The study observed the extension of the depletion zone in the microchannel, analyzed the dimensionless length of depletion zone varying with time and the propagation of the concentration perturbation in the depletion zone.

\section{Experimental apparatus and method}

\subsection{Experimental setup}

The experiments were carried out at the LNM using an inverted fluorescence microscope (type Olympus IX-71) with a $10 \times / 0.3$ lens. The images were recoded by an EMCCD camera (type Andor 885). A DC power supply (using Zolix Instruments Co., Ltd, Model DYY-12C) was used to provide the electrical potential. The range of the experimental operating voltages was $0-100 \mathrm{~V}$ and the maximum output current was $0.6 \mathrm{~mA}$ with output instability below $\pm 0.03 \% / \mathrm{h}$.

\subsection{Chip fabrication}

The hybrid micro/nano-channels used in this study were constructed so as to produce the device shown in Fig. 1 (Kuo et al. 2003). The upper channel on that figure is the source channel. Two poly(dimethylsiloxane) (PDMS) micro-channels were connected together across a polycarbonate nano-porous membrane (PCTE, with polyvinylpyrrolidone (PVP) coating). These membranes were made by a nuclear-etching process. The micro-channels were $100 \pm 0.1 \mu \mathrm{m}$ in width, $20 \pm 0.1 \mu \mathrm{m}$ in depth and $9 \pm 0.02 \mathrm{~mm}$ in length. Hydrophilic PCTE membranes (manufactured by Whatman Corporation) with pore diameters $d=\phi 15$ or $\phi 50 \mathrm{~nm}$ used to separate the two microchannels. It was found from the SEM images that the densities of the $\phi 15 \mathrm{~nm}$ and $\phi 50 \mathrm{~nm}$ pores membranes were about $6 \times 10^{8}$ and $8 \times 10^{8}$ per $\mathrm{cm}^{2}$, corresponding on the porosities of $0.11 \%$ and $1.57 \%$, respectively. The membrane thickness was $6 \mu \mathrm{m}$ for the membrane with $\phi$ $15 \mathrm{~nm}$ pores and $8 \mu \mathrm{m}$ for the membrane with $\phi 50 \mathrm{~nm}$ pores. The thickness error in the membranes was measured to be $\pm 0.8 \mu \mathrm{m}$. The membranes were translucent at the light wave length of interest. Great care was taken in the construction of the hybrid channel to ensure that the two channels were bonded together such that they were orthogonal to each other and that both channels were centered. The PCTE membrane was carefully bonded between the two channels during this construction process. In order to reduce any hydrostatic pressure effects that may arise from the height difference of liquid columns in the reservoirs, relatively large-diameter reservoirs were fabricated. All the tests were conducted with the micro-channels placed horizontally in accord with Fig. 1.

\subsection{Reagents}

$10 \mu \mathrm{M}$ Calcein fluorescence dye was used as the tracer ion in the buffer solution and made visible via a mercury lamp light source. It can be noted that the wave length of the exciting light was $488 \mathrm{~nm}$ while the emitted light was at a wave length of $532 \mathrm{~nm}$. The two different buffer concentrations used in the experiments were specified as:

1. $10 \mathrm{mM}$ (using borax: sodium tetraborate-10-hydrate) at $\mathrm{pH} 9.2$.

2. $10 \mathrm{mM}$ (using a mono-bipotassium phosphate buffer solution-referred to as PBS) at $\mathrm{pH}$ 5.4.

The concentration of the fluorescence was smaller than the concentration of the buffer solution so that the fluorescence only played the role of a tracer indicating the flow development. All the aqueous solutions were prepared with DI water (obtained from Millipore ${ }^{\circledR}$ ).

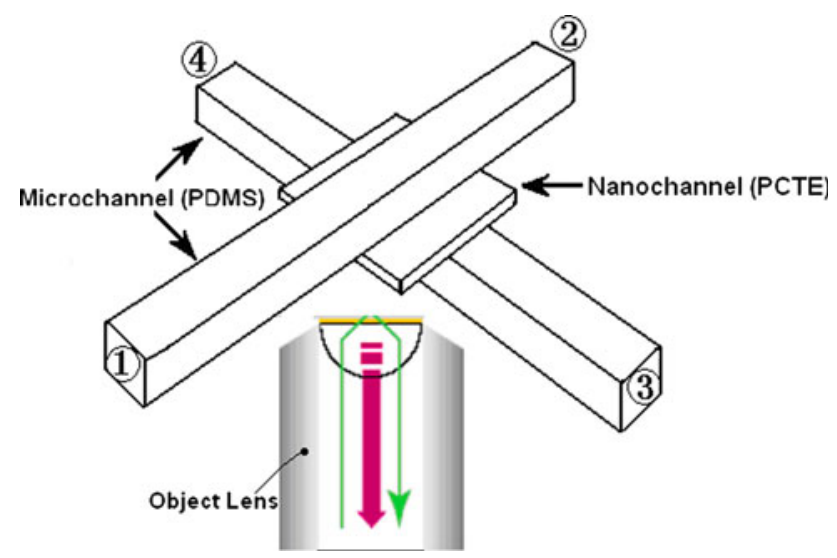

Fig. 1 Schematic diagram of the hybrid micro-/nano-channel used in the experiments 


\subsection{Image processing}

The focal plane of the EMCCD camera was adjusted to view, from vertically below, the upper surface of the nanoporous membrane in all of the experiments reported herein (see Fig. 1). The image size was $(800 \pm 1.6) \times(800 \pm$ 1.6) $\mu \mathrm{m}$. Images were captured with an exposure time of $\Delta t_{\mathrm{p}}=20 \mathrm{~ms}$ and interval time $\Delta t_{1}=0.5 \mathrm{~s}$ for applied voltages of 25 and $50 \mathrm{~V}$. The interval time was $\Delta t_{2}=0.25 \mathrm{~s}$ when the applied voltages were 75 and $100 \mathrm{~V}$. Images from 50 to 100 frames, depending on the applied voltage, were recorded. To avoid the problem of pixel saturation, the image contrast and brightness were carefully adjusted. These considerations fixed the value of the exposure time, $\Delta t_{\mathrm{p}}$, noted above.

\subsection{Experimental procedure}

At the beginning of the experiment, the upper (source) channel (Fig. 2) was filled with fluorescence solution and the bottom (receiving) channel was filled with either pure borax, or the PBS, buffer solution depending upon the required $\mathrm{pH}$ value. Care was taken to exclude all bubbles from the channels. Those chips that were found to suffer leakage during this filling process were rejected. The fluid was at rest at the start of the experiments so that there was no residual motion left over from the filling process. Platinum electrodes were inserted into the reservoirs of the source micro-channel to provide the electrical potential across the PCTE membrane. During the series of experiments, different DC voltages of $25,50,75$ or $100 \mathrm{~V}$ were applied to the reservoirs of the source channel (Fig. 2). The reservoirs of the receiving channel were grounded in all the experiments reported herein. Figure 2 is the schematic of the voltage load way.

\section{Experimental results}

The individual sets of experimental results can be discussed in sequence.
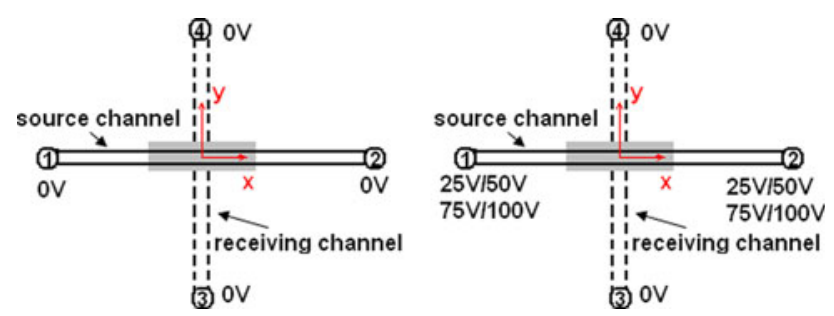

Fig. 2 The configurations of the applied bias at the off (left) and on (right) states
3.1 Experiments with the $\phi 50 \mathrm{~nm}$ diameter nano-porous membrane and $\mathrm{pH} 9.2$

Figure 3 represents the species transport images in the micro-channels at various times. At $t=0 \mathrm{~s}$ the fluorescence was only detected in the source channel (see Fig. 3a, horizontal channel), no fluorescence was found in the receiving channel (vertical channel). When a DC voltage of $25 \mathrm{~V}$ was applied at both ends of the source channel, an ion-depletion zone was seen to form in the source channel. After $2 \mathrm{~s}$ this zone had progressed as shown in Fig. 3b. Meanwhile, some fluorescence ions were found to have migrated to the receiving channel to form an enrichment zone at the cathode side of the nanoporous membrane, again seen in Fig. 3b. At $t=4 \mathrm{~s}$ the length of the iondepletion zone had increased and the fluorescence intensity in ion-enrichment zone had been further enhanced (Fig. 3c). Finally, when $t=6 \mathrm{~s}$, the fluorescence in the source channel had migrated nearly out of image boundary and was only detected in the receiving channel (see Fig. 3d). The boundary of the depletion zone is not like a plunger as pure electroosmosis flow profile but meniscus which defines the convection flow complex.

The information contained in Fig. 3 has been quantified as shown in Fig. 4, which gives the fluorescence intensity, as gray scale values, along the central axis of the source channel as a function of time. On this figure, $x$ represents

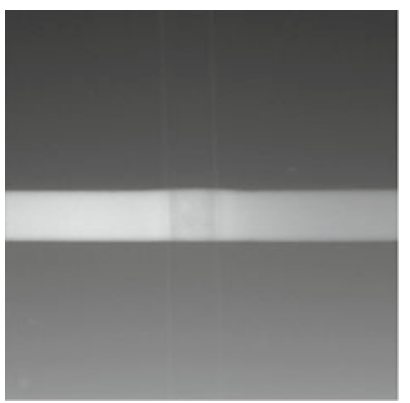

(a)

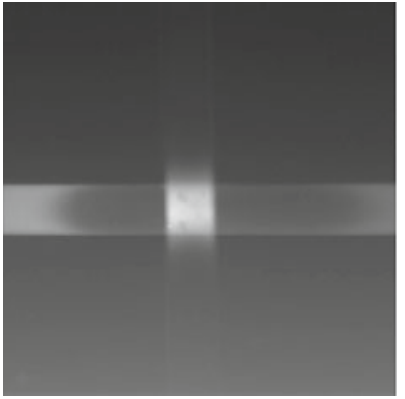

(c)

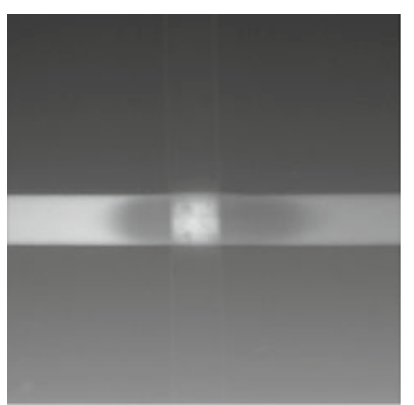

(b)

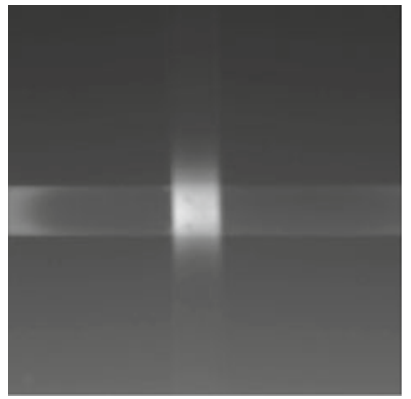

(d)
Fig. 3 The development of Calcein fluorescence using $\phi 50 \mathrm{~nm}$ membrane under a $25 \mathrm{~V}$ potential. a $t=0 \mathrm{~s} ; \mathbf{b} t=2 \mathrm{~s} ; \mathbf{c} t=4 \mathrm{~s}$; d $t=6 \mathrm{~s}$ 


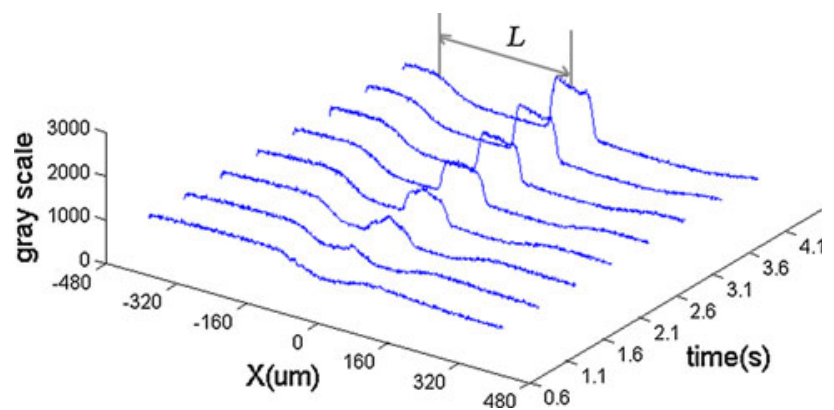

Fig. 4 The fluorescence intensity of the source channel central axis versus time curve under a $25 \mathrm{~V}$ potential $(\phi 50 \mathrm{~nm})$

the central axis of the source channel with zero at the center of cross-section; the time axis is as shown and the vertical axis is the fluorescence intensity presented in gray scale values. The fluorescence signal near the intersection of the hybrid channel is not the information on the source microchannel but signal of the receiving microchannel. This figure clearly shows that, $L$, the ion-depletion zone length increases with the time (over the time interval of the experiment).

In order to study the influence of external potential, in addition to the experiments using a $25 \mathrm{~V}$ potential, experiments with 50,75 , and $100 \mathrm{~V}$ potential were also carried out, respectively. Figure 5 shows the images in the micro-channels at $t=1 \mathrm{~s}$ after loading these different voltages, respectively.

Figure 6 plots the length of the depletion zone in the source channel against the times for different applied voltages (in the range $25-100 \mathrm{~V}$ ). As the voltage increases, the time taken for $L$ to reach the same value was found to decrease. For example, at $25 \mathrm{~V}$, the time taken for $L$ to extend to $280 \mu \mathrm{m}$ was $2.6 \mathrm{~s}$, while with $75 \mathrm{~V}$ the time required for $L$ to extend to $290 \mu \mathrm{m}$ was only $0.95 \mathrm{~s}$.

\subsection{Experiments with $\phi 15 \mathrm{~nm}$ diameter nano-porous membrane and $\mathrm{pH} 9.2$}

To analyzing the influence of pore diameter in the PCTE membrane, a $\phi 15 \mathrm{~nm}$ diameter nano-porous membrane

Fig. 5 The images in the micro-channels with different voltages at same time of $t=1 \mathrm{~s}$. a $50 \mathrm{~V} ; \mathbf{b} 75 \mathrm{~V}$; c $100 \mathrm{~V}$ was also tested. The same basic phenomena were observed as were found for the $\phi 50 \mathrm{~nm}$ membrane. Again at $t=0 \mathrm{~s}$, the fluorescence solution was only contained in the source channel (Fig. 7a). The ion-depletion and ion-enrichment phenomena were found to take place as the loading time increased. That is, the ions are depleted on the anodic side of the nano-porous membrane and accumulated at the cathodic side of the membrane (Fig. 7b, c). Compared to Fig. 3, the time for the case with $\phi 15 \mathrm{~nm}$ nanoporous membrane is more than $14 \mathrm{~s}$ when the depletion length reaches the image boundary, while that is about $6 \mathrm{~s}$ with $\phi$ $50 \mathrm{~nm}$ nanoporous membrane. It means that the rate of the depletion zone evolution is slower with the $\phi 15 \mathrm{~nm}$ pore diameter membrane than it was with the $\phi 50 \mathrm{~nm}$ pore diameter membrane.

The other voltages $(50,75$, and $100 \mathrm{~V})$ were also used in the $\phi 15 \mathrm{~nm}$ nanoporous membrane. Figure 8 is the experimental data points for the evolution of the depletion zone as a function of the loading time under the fourth external electric fields. As the voltage increases, the time taken for $L$ to reach the same value was found to decrease.

\subsection{Experiments with $\phi 50 \mathrm{~nm}$ diameter nano-porous membrane and $\mathrm{pH} 5.4$}

As we know, if the $\mathrm{pH}$ value changed, the surface charge density would change. And then the bulk velocity would change, which may affect the ion-depletion extension. Refer to Kirby and Hasselbrink (2004), the PDMS microchannel display a negative surface at $\mathrm{pH} 9.2$ and the surface charge densities decreases at the lower $\mathrm{pH}$ values $(\mathrm{pH}$ 5.4). Moreover, the PCTE membranes are coated with PVP, which would be protonated at $\mathrm{pH} 9.2$ (Kuo et al. 2001), the surface of membrane is positively charged. It will become larger at the lower $\mathrm{pH}$ values. Figure 9 shows the species transport images at the solution of $\mathrm{pH}$ 5.4.

Figure 10 shows how the depletion zone length varies with the loading time under two buffer $\mathrm{pH}$ values at an applied voltage of $25 \mathrm{~V}$. When the buffer $\mathrm{pH}$ value is small, the depletion zone extended more slowly than it did for the higher buffer $\mathrm{pH}$ value. For example, at $25 \mathrm{~V}$ and

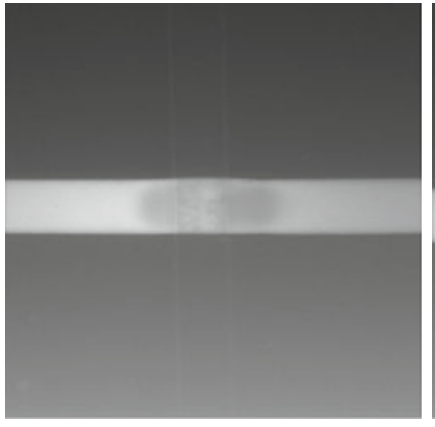

(a)

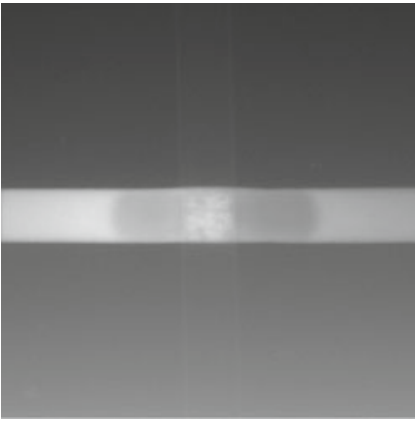

(b)

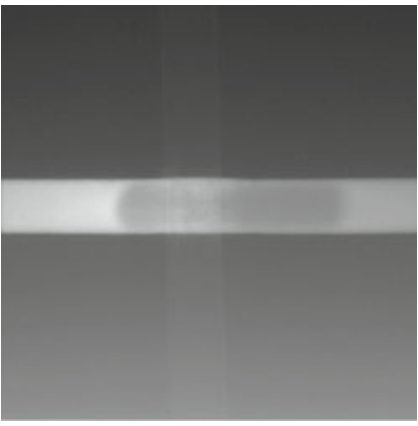

(c) 


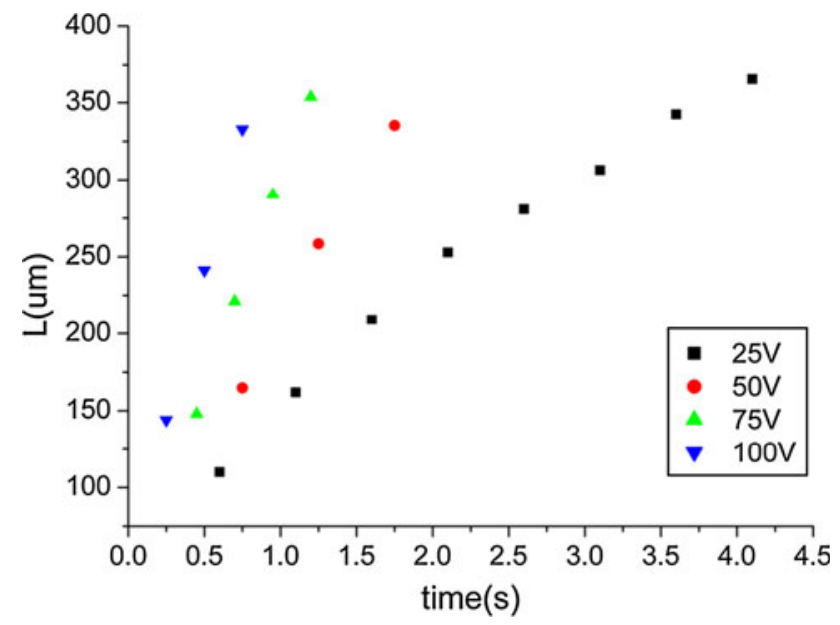

Fig. 6 The curves of the depletion zone length with the time and applied voltages $(\phi 50 \mathrm{~nm}, \mathrm{pH} 9.2)$

the buffer $\mathrm{pH}$ value of 9.2, the evolution length was about $280 \mu \mathrm{m}$ when $t=2.6 \mathrm{~s}$, while for the $\mathrm{pH}$ value of 5.4 this evolution length was only $169 \mu \mathrm{m}$ for the same time.

\section{Discussions of the results}

\subsection{Non-dimensional analysis}

The data shown in Figs. 6, 8, and 10 represent the change in the depletion zone length with time for different voltages, pore diameters and buffer $\mathrm{pH}$ values. Create a normalization for the ion-depletion zone length and the time interval for the motion based upon the Smoluchowski slip velocity $U=-{ }^{\varepsilon E \zeta} / \eta$ (Smoluchowski 1916) of the microchannel, and the geometry of the device. Then define:

$L^{*}=\frac{L}{L_{\mathrm{m}}}, \quad t^{*}=\left[\frac{d}{\lambda}\right] \cdot\left|-\frac{\varepsilon_{0} \varepsilon_{\mathrm{r}} \zeta E}{\eta}\right| \cdot\left[\frac{t}{L_{\mathrm{n}}}\right]$

where $L_{\mathrm{m}}$ denotes the micro-channel length, $d$ the nanoporous diameter, $L_{\mathrm{n}}$ the nano-porous membrane thickness, $\lambda$ the double layer thickness, $\eta$ represents the viscosity

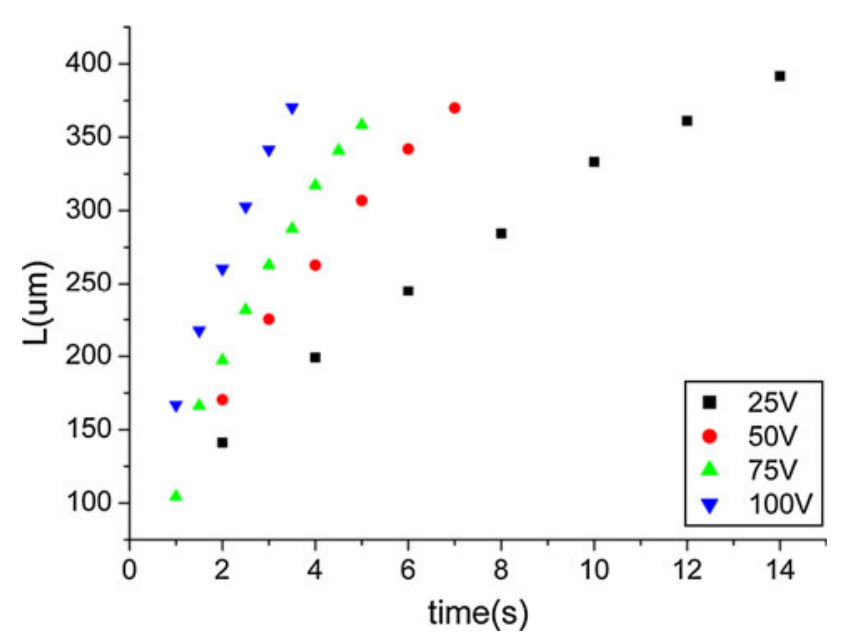

Fig. 8 The curves of the depletion zone length with the time and applied voltages $(\phi 15 \mathrm{~nm}, \mathrm{pH} 9.2)$

coefficient of the fluid, $E$ is the electric field strength. $\varepsilon_{0}$ and $\varepsilon_{\mathrm{r}}(=80)$ are the vacuum permittivity and dielectric constant, respectively. Also $\zeta$ represents the zeta potential for the wall of the microchannel. Here, we use that the value of $\zeta$ is $-30 \mathrm{mV}$ for the case when buffer solution had $\mathrm{pH} 9.2$ while the value is $-15 \mathrm{mV}$ for the $\mathrm{pH} 5.4$ case.

Figure 11 is a summary of the data presented in Figs. 6, 8, and 10. And it can be seen from Fig. 11 that the dimensionless evolution length varies linearly with nondimensional time for the interval $t^{*}<220$. For larger $t^{*}$ values there is a non-linear variation and the formula $L^{*} \sim \sqrt{t^{*}}$ is observed to be appropriate.

Therefore, the extension velocity of the depletion boundary, $V$ is defined as,

$V=L / t=\frac{L^{*}}{t^{*}} \cdot \frac{L_{\mathrm{m}}}{L_{\mathrm{n}}} \cdot \frac{d}{\lambda} \cdot \frac{\varepsilon_{0} \varepsilon_{\mathrm{r}} E|\zeta|}{\eta}$

For small time, $V \sim \frac{L_{\mathrm{m}}}{L_{\mathrm{n}}} \cdot \frac{d}{\lambda} \cdot \frac{\varepsilon_{0} \varepsilon_{\mathrm{r}} E|\zeta|}{\eta}$, while for the large time, $V$ is proportion to the reciprocal of square root of the loading time with $V \sim \sqrt{\frac{L_{\mathrm{m}}^{2}}{L_{\mathrm{n}}} \cdot \frac{d}{\lambda} \cdot \frac{\varepsilon_{0} \varepsilon_{\mathrm{r}} E|\zeta|}{\eta}}$. When the other parameters fixed, the extension velocity satisfies the
Fig. 7 The development of Calcein fluorescence for the $\phi$ $15 \mathrm{~nm}$ membrane under a $25 \mathrm{~V}$ potential. a $t=0 \mathrm{~s} ; \mathbf{b} t=2 \mathrm{~s}$; c $t=14 \mathrm{~s}$



(a)

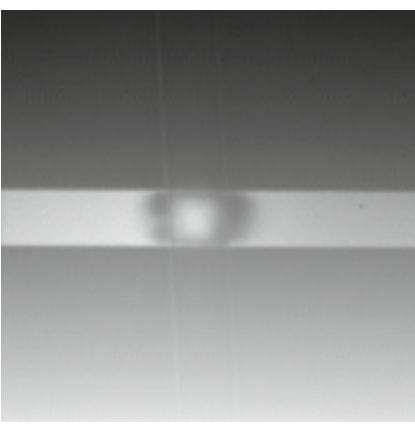

(b)

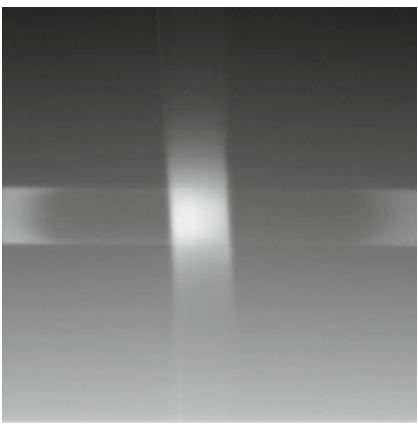

(c) 
Fig. 9 The images of Calcein fluorescence using $\phi 50 \mathrm{~nm}$ membrane under a $25 \mathrm{~V}$ potential at $\mathrm{pH} 5.4$. a $t=0 \mathrm{~s}$ b $t=2 \mathrm{~s} ; \mathbf{c} t=4 \mathrm{~s}$

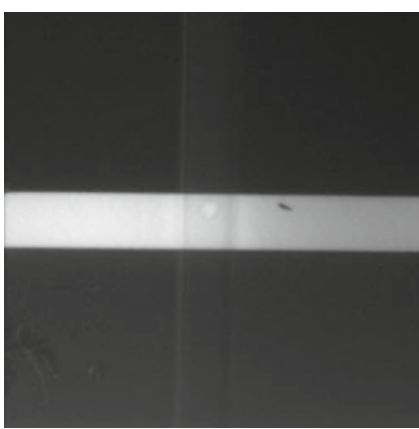

(a)

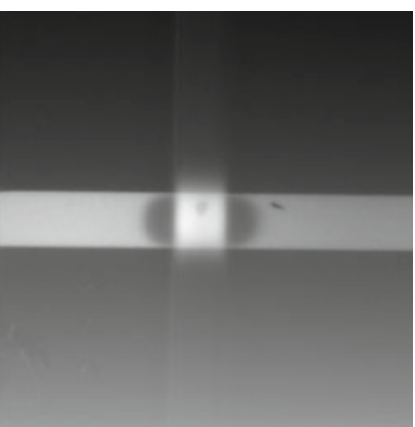

(b)

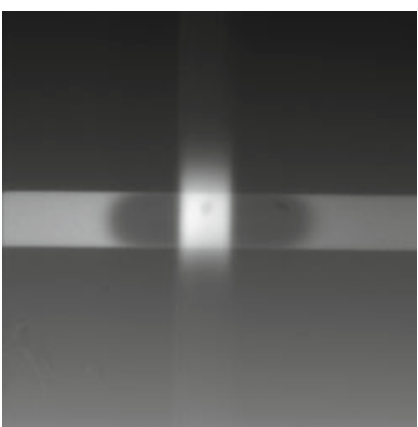

(c) following relations: $V \sim \sqrt{E}, V \sim \sqrt{d}$, and $V \sim \sqrt{|\zeta|}$, that is, the extension velocity increases as the parameters of electric field strength $E$, nano-pore size, and $\mathrm{pH}$ value increase, respectively. This is consistent with the data shown in Figs. 6, 8, and 10: when $E$ increases, the bulk velocity becomes larger and the depletion extension is more rapid (Figs. 6 and 8); since $\mathrm{pH}$ value is related with the zeta potential, when $\mathrm{pH}$ value increases, bulk velocity also becomes larger and depletion extension is more rapid (Fig. 10); as pore size d increases, the depletion extension also seems to be more rapid (compared Figs. 6 and 8). Moreover, if nano-pore size and zeta potential are constant during loading time, $V$ changes only related with $E$ at beginning and $\sqrt{E}$ for large time.

4.2 The propagation of the concentration perturbation in the depletion zone

According to flow visualization shown in Sect. 3, we observed the time extending of the ion-depletion zone, and in Sect. 4.1, we discussed the dimensionless laws of the depletion length with the loading time under the applied

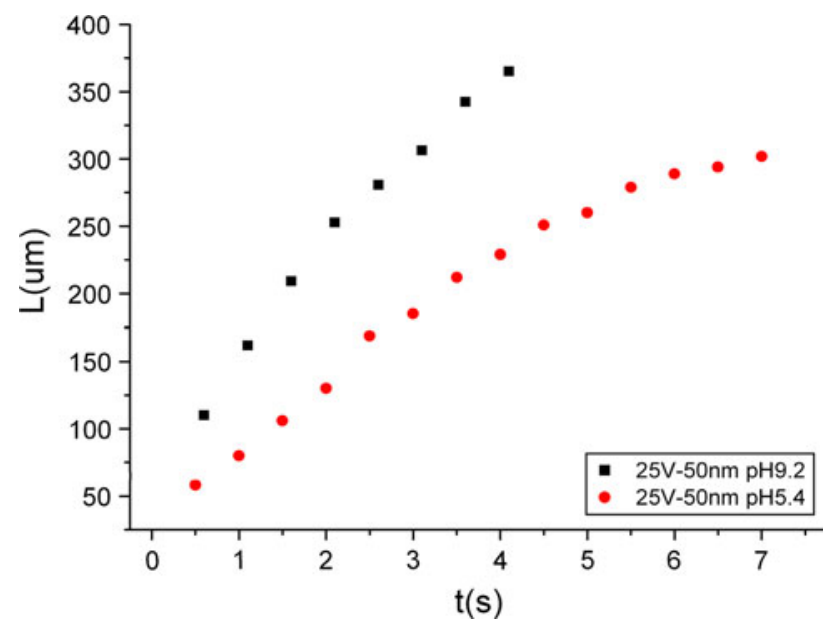

Fig. 10 Change of the length of the depletion zone with the loading time under different $\mathrm{pH}(\phi 50 \mathrm{~nm}, 25 \mathrm{~V})$ electric strength, nanopore size of the membrane and the $\mathrm{pH}$ value of buffer solution.

But the concentration perturbation is not mentioned. From Fig. 3, we also noted that the boundary of depletion zone divided lower and higher concentrations and indicated the strong concentration gradient. As depletion zone is extended, the concentration perturbation is propagated. Here, we introduce the Nernst-Planck equation to investigate the concentration changes in the depletion zone. From Fig. 4, we get the concentration distribution on the left position of the central axis in the source microchannel with different loading times (Fig. 12). If the position
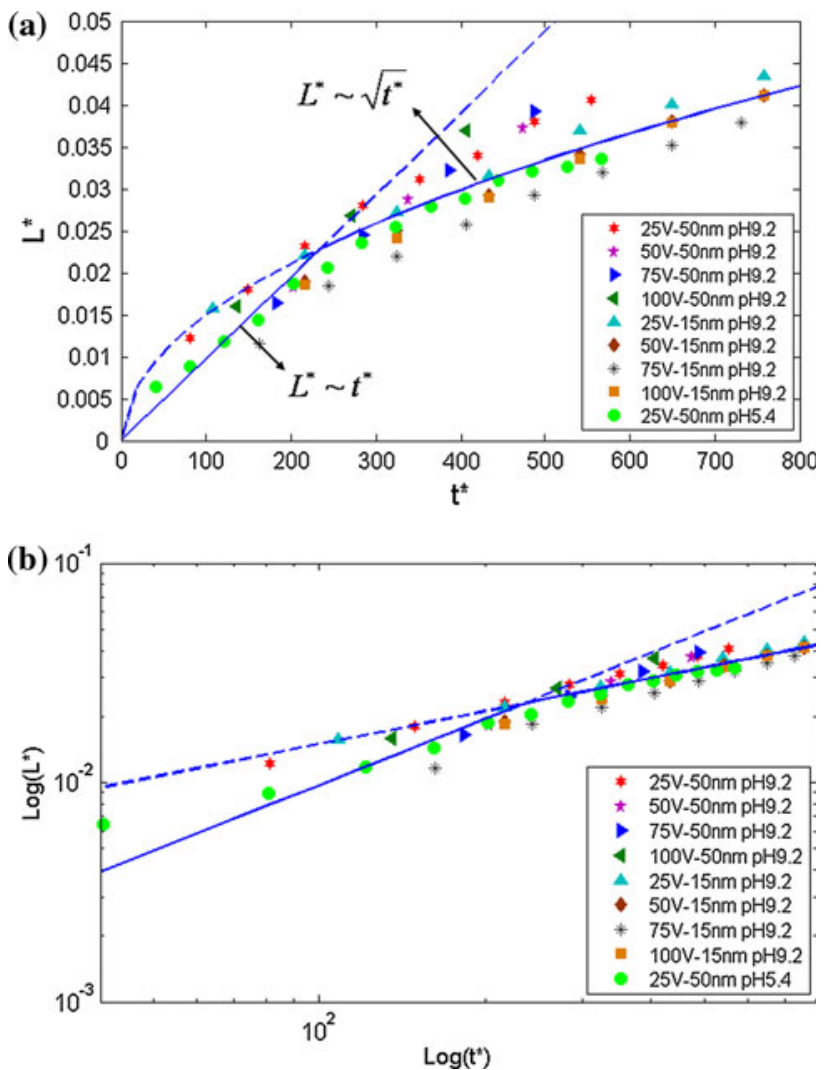

Fig. 11 The extension of dimensionless depletion length with the dimensionless loading time values (a), its $\log / \log$ plot (b) 
$X$ fixed, we may see the concentration changes $(\Delta c)$ corresponding to the different time changes $(\Delta t)$. Figure 13 shows, the time rate of the concentration change is approximately linear at a certain moments (for example $t=1.6-2.6$ for $x=-160 \mu \mathrm{m})$ and then becomes nonlinear.

The analysis of the concentration perturbation in the microchannel flow can be continued from a more theoretical point of view. Start by adopting the ion conservation equation with the Nernst-Planck flux density (Probstein 2003) in the form:

$\frac{\partial c_{i}}{\partial t}=-\nabla \cdot\left(-D_{i} \cdot \nabla c_{i}-z_{i} c_{i} F \mu_{i} \nabla \psi+c_{i} \vec{u}\right)$

where $c_{i}$ is the concentration for ionic species $i(i=1$ and 2 denotes the positive and negative ions, respectively), $z_{i}$ is the valence, and $D_{i}$ is the diffusion coefficient. In addition $F$ represents the Faraday constant and $\mu_{i}\left(=\frac{D_{i}}{R T}\right)$ is the ionic mobility. $\vec{u}$ is the velocity vector of the bulk flow, and $\psi$ is the local electrical potential. The three terms on the righthand side of Eq. 3 define the fluxes due to diffusion, electrical migration, and convection, respectively.

Introduce the Poisson equation $\nabla^{2} \psi=-\frac{\rho_{\mathrm{e}}}{\varepsilon}(\varepsilon$ is the permittivity) and the definition of the charge density $\rho_{\mathrm{e}}=F \sum z_{i} c_{i}$. For the negative ions, Eq. 3 becomes:

$\frac{\partial c_{2}}{\partial t}=D_{2} \nabla \cdot\left(\nabla c_{2}\right)-z_{2} F \mu_{2} E \cdot \nabla c_{2}-\frac{z_{2} c_{2} F^{2} \mu_{2} \sum z_{i} c_{i}}{\varepsilon}$

$-\vec{u} \cdot \nabla c_{2}-c_{2} \nabla \cdot \vec{u}$

Now, because of the continuous medium hypothesis $\nabla \cdot \vec{u}=0$ and the fifth term on the right-hand side of Eq. 4 vanishes identically. A simple phenomenological one-dimensional model of the motion within the source microchannel can be constructed. This model is not intended as a theory of such flows but only as a model to



Fig. 13 The curves of the gray scale with different time at the positions of $X=-160 \mu \mathrm{m}, X=-200 \mu \mathrm{m}, X=-240 \mu \mathrm{m}(25 \mathrm{~V}$, $\phi 50 \mathrm{~nm}, \mathrm{pH}$ 9.2)

identify the main physical processes involved. The model is limited to discussing the evolution of the length $L$ that was obtained in the experimental results presented above. In such a one-dimensional model, the continuity equation reduces to the statement $d u_{x} / d x=0$. Hence $u_{x}=u_{x}(t)$ only. Here, $u_{x}$ is the $x$ component of the fluid bulk velocity vector $\vec{u}$, a quantity which must be obtained from the NavierStokes equations. The $\vec{u}$ field is more general than this onedimensional form in the real flow. Equation 4 reduces to:

$$
\begin{aligned}
\frac{\partial c_{2}}{\partial t}+\left(u_{x}+z_{2} F \mu_{2} E\right) \frac{\partial c_{2}}{\partial x}= & D_{2} \nabla \cdot\left(\nabla c_{2}\right) \\
& -\frac{z_{2} c_{2} F^{2} \mu_{2} \sum z_{i} c_{i}}{\varepsilon}
\end{aligned}
$$

Compared with the third term on the left and the first term on the right, $\frac{z_{2} F E D_{2} / R T}{D_{2} / L_{\mathrm{m}}}$, is of the order $O\left(10^{2}\right)$. Therefore, the diffusion term can be neglected for the initial motion. In addition, owing to the buffer solution
Fig. 12 The spatiotemporal changes of the gray scale in the left part of the microchannel (25 V, $\phi 50 \mathrm{~nm}, \mathrm{pH} 9.2)$

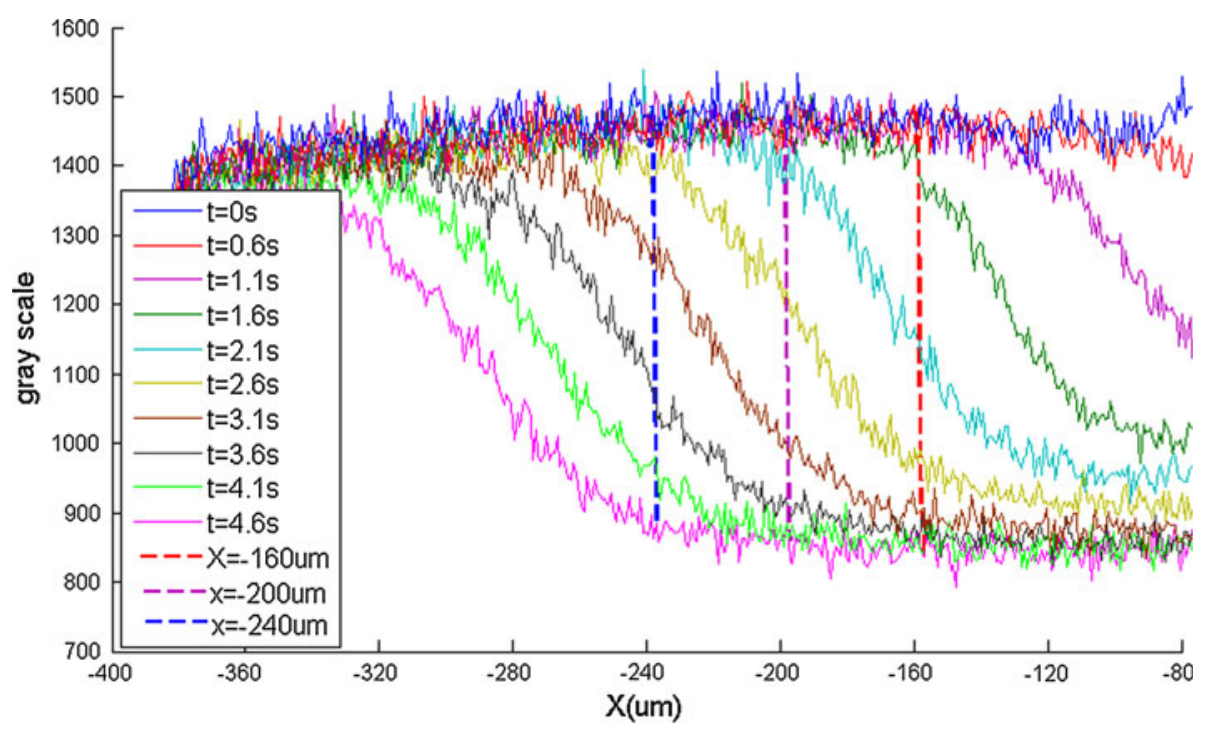


being, for small time, essentially electrical neutral in the microchannel, the second term on the right-hand side of Eq. 5 can be ignored as a first approximation. This gives:

$\frac{\partial c_{2}}{\partial t}+\left(u_{x}+z_{2} F \mu_{2} E\right) \frac{\partial c_{2}}{\partial x}=0$

a simple wave equation for the concentration. Equation 6 shows that, for short times, if $u_{x}$ and $\mathrm{E}$ are taken to be approximately constant, the propagation speed of the concentration perturbation must change linearly with time. Specifically Eq. 6 implies, for short times, that the concentration has the form: $c_{2}=f\left[x \pm\left(u_{x}+z_{2} F \mu_{2} E\right) t\right]$ as two waves propagating in opposite directions along the microchannel. Here $c_{2}=f[\cdot]$ presents the extension fronts of depletion zone for short time as two solid lines shown in Fig. 14. The extent, in time, of such linear motion depends upon the local flow parameters such as $E$ or the $\mathrm{pH}$ value of the fluid.

Denote by $V_{c}=u_{x}+z_{2} F \mu_{2} E$, the concentration perturbation speed along the source channel. For short time, this initial speed is unchanged as long as the approximations, $u_{x}$ and $\mathrm{E}$ constant, hold. $V_{c}$ seems as same as the velocity $V$ given in Eq. 2, but only for short time when $L$ extends linearly. This constant propagation speed increases as the electric strength increases which is clearly shown in Figs. 6 and 8. The zeta potential of the microchannel and the ion valence will decreases as the $\mathrm{pH}$ value of the buffer solution decreases, so the concentration perturbation speed will also decrease (see Fig. 10).

After this initial time interval, the non-linear effects must be included as Eq. 5 and the graph of $L$ against time is only linear when the correlation for $t^{*}<220$ of Fig. 11 applies. It is not evident, from the above discussion, why the variation of $L$ should change from a linear to a square root variation at this value of time. It is clear that for larger time $u_{x}$ is no longer a constant and $E$ also changes as the ion concentration changes. The divergence of the NavierStokes equation, which produces a Poisson equation for the pressure, implies that at long time the induced pressure distribution follows from the electric field and the ion

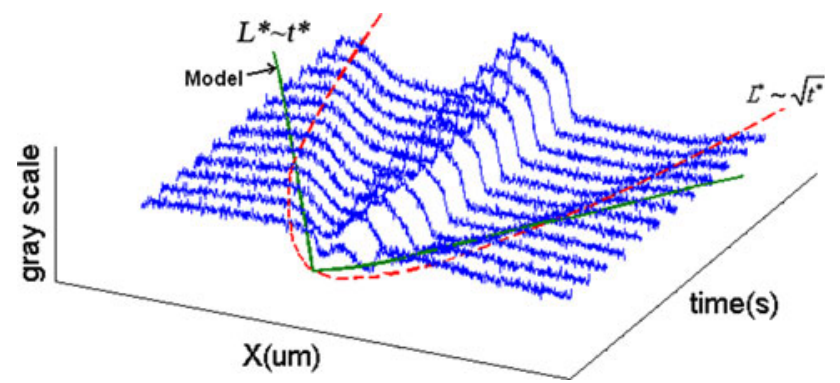

Fig. 14 Schematic diagram of dimensionless law (from Sect. 4.1) and linear approximate phenomenological model (Sect. 4.2) for extension of depletion zone concentration as shown from experiments given by Silber$\mathrm{Li}$ et al. (2011), or the complete calculation using a numerical simulation of Jin et al. (2007).

This model is only proposed for short time so that the approximations made above are reasonable. It is well known that for long time there is the possibility of vortex formation in these devices.

\section{Conclusions}

The present paper has described the electro-kinetic flow behavior of Calcein fluorescence solution under different applied voltages (namely $25,50,75$, and $100 \mathrm{~V}$ ). In addition, the effect of nano-porous membrane diameter (using $\phi 15 \mathrm{~nm}$ and $\phi 50 \mathrm{~nm}$ ) was examined. Two different buffer solutions, with $\mathrm{pH}$ values of $\mathrm{pH} 5.4$ and 9.2, were also used in the study. The objective was to observe and measure the extension of the depletion zone and the propagation of the concentration perturbation. The main results included:

1. The experiments indicate that the ion-depletion zone extends rapidly with the electrical field strength, the buffer solution $\mathrm{pH}$ value and the pore diameter of the nano-porous membrane increases, respectively: when the $\phi 50 \mathrm{~nm}$ nano-porous membrane was used in the $\mathrm{pH} 9.2$ buffer solution, the time taken for the depletion zone length, $L$, to extend to $280 \mu \mathrm{m}$ was $2.6 \mathrm{~s}$ at $25 \mathrm{~V}$. However, the time required for $L$ to extend to $290 \mu \mathrm{m}$ was only $0.95 \mathrm{~s}$ at $75 \mathrm{~V}$; If the buffer solution was replaced by the one of $\mathrm{pH} 5.4$, the evolution length was $169 \mu \mathrm{m}$ for $t=2.6 \mathrm{~s}$ at $25 \mathrm{~V}$, the depletion zone extends slowly. The experiments also showed that when the $\phi 15 \mathrm{~nm}$ pore diameter was used, the time $2.6 \mathrm{~s}$ can only cause the depletion boundary to extend to $166 \mu \mathrm{m}$ at $25 \mathrm{~V}$. Therefore, the extending velocity had decreased, compared with that for the $\phi 50 \mathrm{~nm}$ nano-porous membrane.

2. For summary, we carried out the non-dimensional analysis on the extending length of the depletion zone and the loading time, it contains two parts: linear region for short time and non-linear region for long time with the relation of $L^{*} \sim \sqrt{t^{*}}$.

3. From the Nernst-Planck equation, one-dimensional phenomenological model is introduced to show the propagation of concentration in the depletion zone. The propagation of the concentration perturbation goes through two stages: linear region for short time and non-linear region for long time. In the short time, the propagation speed of concentration perturbation has the form of $c_{2}=f\left[x \pm\left(u_{x}+z_{2} F \mu_{2} E\right) t\right]$, and the propagation speed is as $V_{c}=u_{x}+z_{2} F \mu_{2} E$ which keeps a constant for the initial time. After that the 
internal electric field and the bulk velocity is no longer a constant, the propagation speed of concentration perturbation then changes with the time depended upon the nonlinear wave equation.

Although we have presented the extension of the depletion zone with time and analyzed the propagation of concentration perturbation, it is short for the depletion formation related with ion transport in nanochannels. In addition, the porosities of the nanoporous membranes are not the same for different pore size, it maybe influence the analysis about the pore size. Also, for ion-enrichment observed in the experiment should be analyzed in the further research.

Acknowledgments The authors wish to acknowledge, with thanks, the financial support of the National Basic Research Program of China (2007CB714501), Knowledge Innovation Program of CAS (KJCX2-YW-H18) and the National Natural Science Foundation of China (10872203). The authors thank Trevor H. Moulden for useful discussions.

\section{References}

Datta A, Gangopadhyay S, Temkin H, Pu QS, Liu SR (2006) Nanofluidic channels by anodic bonding of amorphous silicon to glass to study ion-accumulation and ion-depletion effect. Talanta 68:659-665

Garcia AL, Ista LK, Petsev DN, O'Brien MJ, Bisong P, Mammoli AA, Brueck SRJ, Lopez GP (2005) Electrokinetic molecular separation in nanoscale fluidic channels. Lab Chip 5:1271-1276

Griffiths SK, Nilson RH (2006) Charged species transport, separation, and dispersion in nanoscale channels: autogenous electric fieldflow fractionation. Anal Chem 78:8134-8141

Huang K-D, Yang R-J (2008) Formation of ionic depletion/enrichment zones in a hybrid micro-/nano-channel. Microfluid Nanofluid 5:631-638

Jin X, Joseph S, Gatimu EN, Bohn PW, Aluru NR (2007) Induced electrokinetic transport in micro-nanofluidic interconnect. Langmuir 23:13209-13222
Kim SJ, Wang YC, Lee JH, Jang H, Han J (2007) Concentration polarization and nonlinear electrokinetic flow near a nanofluidic channel. Phys Rev Lett 99:044501

Kirby BJ, Hasselbrink EF Jr (2004) Zeta potential of microfluidic substrates: 2. Data for polymers. Electrophoresis 25:203-213

Kuo TC, Sloan LA, Sweedler JV, Bohn PW (2001) Manipulating molecular transport through nanoporous by control of electrokinetic flow: effect of surface charge density and Debye length. Langmuir 17:6298-6303

Kuo TC, Bohn PW et al (2003) Hybrid three-dimensional nanofluidicmicrofluidic devices using molecular gates. Sens Actuators A 102:223-233

Lee JH, Song Y-A, Han J (2008) Multiplexed proteomic sample preconcentration device using surface-patterned ion-selective membrane. Lab Chip 8:596-601

Plecis A, Schoch RB, Renaud P (2005) Ionic transport phenomena in nanofluidics: experimental and theoretical study of the exclusion-enrichment effect on a chip. Nano Lett 5:1147-1155

Probstein RF (2003) Physicochemical hydrodynamics, 2nd edn. Wiley Interscience, New York

$\mathrm{Pu}$ Q, Yun J, Temkin H, Liu S (2004) Ion-enrichment and iondepletion effect of nanochannel structures. Nano Lett 4:10991103

Silber-Li ZH, Kong GP, Yu Q, Moulden TH (2011) Observation of vorticity generation in micro/nano-channel flows subject to an electric field. In: Proceedings of the 11th Asian symposium on visualization, Niigata, Japan

Smoluchowski MV (1916) Z Phys Chem 92:129

Song S, Singh AK (2006) On-chip sample preconcentration for integrated microfluidic analysis. Anal Bioanal Chem 384:41-43

Wang YC, Stevens AL, Han J (2005) Million-fold preconcentration of proteins and peptides by nanofluidic filter. Anal Chem 77:42934299

Wang KG, Yue SL, Wang L, Jin AZ, Gu CZ, Wang PY, Feng YC, Wang YC, Niu HB (2006) Manipulating DNA molecules in nanofluidic channels. Microfluid Nanofluid 2:85-88

Yossifon G, Chang H-C (2008) Selection of nonequilibrium overlimiting currents universal depletion layer formation dynamics and vortex instability. Phys Rev Lett 101:254501

Zangle TA, Mani A, Santiago JG (2009) On the propagation of concentration polarization from microchannel-nanochannel interfaces part II: numerical and experimental study. Langmuir 25:3909-3916 